\title{
Bootstrap, An Alternative to Monte Carlo Simulation
}

Indexing term: Monte Carlo simulation, bootstrap, statistical estimate.

Abstract: Monte Carlo simulation is a commonly used technique for circuit analysis, but is computationally expensive. The bootstrap method can save simulation time and retain the desired accuracy.

\section{Introduction}

Monte Carlo simulation has been widely applied to the electronics design and analysis, such as testing, fault detection, sensitivity analysis, production discrimination, etc. Generally speaking, the expensive computational cost of Monte Carlo simulation is a bottleneck in most electronics design and analysis. We therefore propose the Bootstrap method to reduce this computational cost. The accuracy of the approach is demonstrated by comparing the mean and variance obtained from 100 Monte Carlo fault simulation runs of an opamp with a reduced bootstrap sample using only 10 runs.

\section{Bootstrap method}

The Bootstrap method is a well-established robust statistical methodology [1]. Although bootstrap has been widely employed in social sciences [2] and signal processing [3], there are no reports of applying Bootstrap to electronics design. The basic principle of Bootstrap is the random replication of the original sample. Given a desired statistical estimate, $S$, based on a sample $\mathbf{x}$, where,

$$
\mathbf{x}=\left\{x_{1}, x_{2}, \cdots, x_{i}, \cdots, x_{n}\right\}
$$

the first step of the Bootstrap method is to generate a bootstrap sample,

$$
\mathbf{x}^{B}=\left\{\mathbf{x}^{1}, \mathbf{x}^{2}, \cdots, \mathbf{x}^{b}, \cdots, \mathbf{x}^{m}\right\}
$$

where,

$$
\mathbf{x}^{b}=\left\{x_{1}^{b}, x_{2}^{b}, \cdots, x_{n}^{b}\right\}
$$

$\mathbf{x}^{\mathrm{b}}$ has the same dimension as $\mathbf{x}$ and the elements of $\mathbf{x}^{\mathrm{b}}$ are randomly drawn from the original sample. For example, if we have a set $\{1.0,3.1,9.2,6.7\}$, one of the bootstrap samples might be $\{1.0,3.1,3.1,6.7\}$. From $\mathbf{x}^{\mathrm{B}}$, a series of new statistical estimates can be calculated as

$$
\mathbf{S}^{B}=\left\{S^{1}, S^{2}, \cdots, S^{m}\right\} .
$$

A robust estimate of $\mathrm{S}$ is then

$$
S^{*}=E\left(S^{b}\right)
$$

This is particularly effective when the original sample is expensive or obtaining it is difficult.

\section{Desirable properties of the bootstrap method}

The Bootstrap has at least two good asymptotic properties for statistical estimation [1]. The first is the asymptotic property of the estimate. When the number of bootstrap samples becomes larger, the confidence interval will tend to converge. The second is the asymptotic property of distribution. Even using a small number of runs of a Monte Carlo simulation, bootstrap samples show an asymptotic normal distribution. Fig. 1 shows the asymptotic property of the estimate, where the horizontal axis enumerates the bootstrap samples and the vertical axis indicates the estimated values. The solid line represents the estimate and the dotted lines are the confidence interval. Fig. 2 gives an example of asymptotic property of the distribution, where the number of bootstrap samples is 1,000 from 5 runs of a Monte Carlo simulation.

\section{Experimental results}

An example of the use of Monte Carlo analysis is in analogue fault simulation [4]. Monte Carlo analysis is used to derive the mean and variance of fault-free and faulty circuit responses subject to process parameter deviations. The responses are then compared and the effectiveness of the stimulus evaluated. At least 30 Monte Carlo simulations have to be done of both the fault-free circuit and of each faulty circuit to achieve the desired accuracy. However the computational cost of Monte Carlo simulation in SPICE is high. For example, a cascode op-amp circuit containing an opamp with 20 transistors was fault simulated. In total there were 92 faults. 100 runs of a Monte Carlo simulation of these faults using HSPICE needed 20 hours of CPU time on a $170 \mathrm{MHz}$ UltraSPARC. 
A bootstrap sample was collected from 10 of these Monte Carlo simulation runs to compare the accuracy of the bootstrap method. We refer to the HSPICE results as the original samples. A statistical estimate of the mean and variance was derived from each bootstrap sample and the final robust estimate was derived from these estimates of various bootstrap samples.

Fig. 3 shows how the errors between the bootstrap estimate and the standard estimate of the distribution using 100 Monte Carlo runs. The filled patterns represent the errors between the bootstrap estimate and the standard estimate of mean. The open patterns show the errors between the bootstrap estimate and the standard estimate of variance. The horizontal axis indicates the errors and the vertical axis denotes the percentage error. It can be seen that most errors of the mean estimate are less than $10 \%$ and the most errors of the variance estimate are within $30 \%$. Thus the bootstrap estimate of the mean and variance needs 10 runs of Monte Carlo simulation to achieve the same accuracy as the standard estimate based on 100 runs of Monte Carlo simulation.

\section{Conclusions}

The benefit of the Bootstrap method has been demonstrated. It is believed that applying the Bootstrap technique will benefit most areas of electronics analysis, such as fault detection, sensitivity analysis and production discrimination.

\section{References}

1 Efron, B. "Bootstrap methods: another look at the jackknife", The Annals of Statistics, Vol. 7, pp. 1-26, 1979.

2 Efron, B. and Tibshirani, R. J. An Introduction to the bootstrap, London: Chapman and Hall, 1993.

3 Zoubir, A. M. and Boashash, B. "The bootstrap and its application in signal processing", IEEE Signal Processing Magazine, January, pp. 56-76, 1998.

4. Spinks, S.J., Chalk, C.D., Zwolinski, M.and Bell, I.M. "Generation and Verification of Tests for Analogue Circuits Subject to Process Parameter Deviations", IEEE International Symposium on Defect and Fault Tolerance in VLSI Systems, October 20-22, 1997, Paris, France.

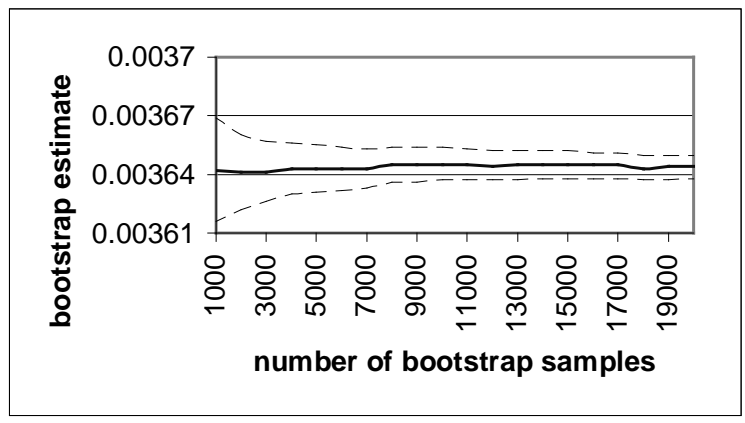

Fig. 1 Asymptotic property of estimate

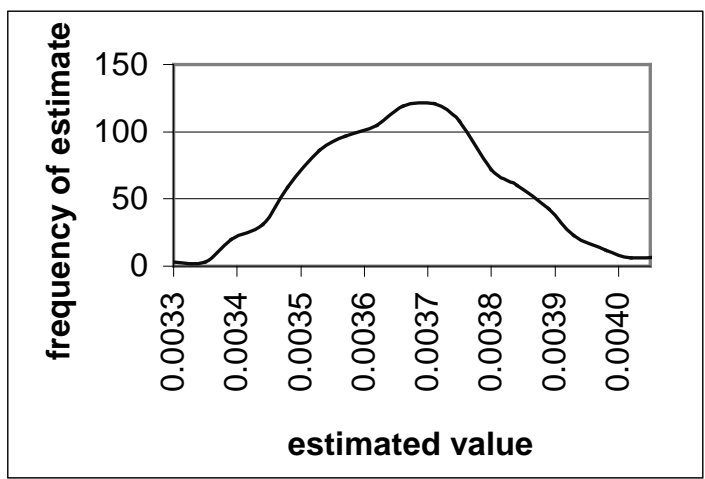

Fig. 2 Asymptotic property of the distribution 


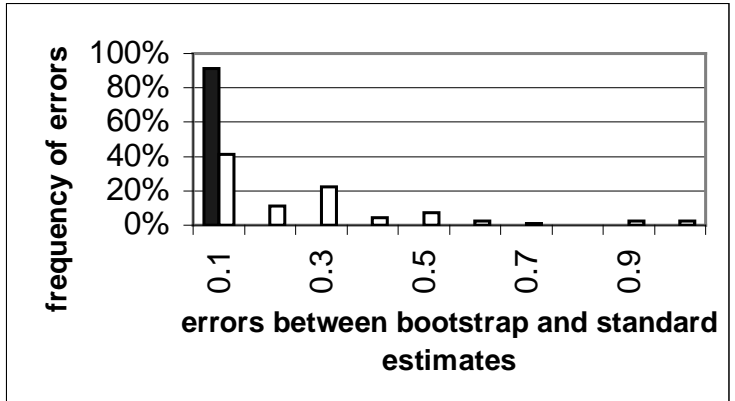

Fig. 3 Distribution of errors between bootstrap and standard estimates 